\title{
Micro, Meso and Macro Porous Materials on Medicine
}

\author{
Victor Solano-Umaña1, José Roberto Vega-Baudrit ${ }^{2}$ \\ ${ }^{1}$ Environmental Management Master Degree, Senior Chemist Hologic Surgical Products, DOCINADE Science \\ PhD Student, San José, Costa Rica \\ ${ }^{2} \mathrm{PhD}$, National Nanotechnology Laboratory (LANOTEC-CeNAT-CONARE), San José, Costa Rica \\ Email: Victor.solano@hologic.com, jvegab@gmail.com
}

Received 4 August 2015; accepted 5 October 2015; published 8 October 2015

Copyright (C) 2015 by authors and Scientific Research Publishing Inc.

This work is licensed under the Creative Commons Attribution International License (CC BY). http://creativecommons.org/licenses/by/4.0/

(c) (i) Open Access

\begin{abstract}
An enormous development is experimented on the porous materials. The porous was considered a defect on solid materials some years ago, but right now, this defect is an advantage, based on the properties obtained from a micro and mesoporous materials. Microporous, mesoporous and macroporous materials with an uniformed pore distribution offer new properties, such as absorption, adsorption, exchange separation, and catalysis of different compounds, also they can play different roles as hosts for a nanocomposite materials to modify or improve their properties. Today, the structural types of open framework porous compounds have rapidly increased by their unique structural properties, porous size window and accessible void space are critical factors on a medical application.
\end{abstract}

\section{Keywords}

\section{Microporous, Mesoporous, Macro Porous}

\section{Introduction}

Immense research during the last decade has been devoted to adjust the morphology (the size and shape) of solids at various length scales from the nanometer to the micrometer scale [1]. The porous materials research has been done with a variety of pore sizes, shapes and densities, but the crucial feature is the increase in the material surface, which helps improve property absorbent and adsorbent properties [2] [3]. Modifying the surface of materials, you can obtain materials with different porous size.

Porous materials are classified by the pore size; the International Union of Pure and Applied Chemistry (IUPAC) classify the porous materials in: 
- Microporous, materials have pore diameters of less than $2 \mathrm{~nm}$;

- Mesoporous, materials have pore diameters between $2 \mathrm{~nm}$ and $50 \mathrm{~nm}$;

- Macro porous, materials have pore diameters of greater than $50 \mathrm{~nm}$.

Microporous, Mesoporous and Macro porous materials can be manufactured using many substances such as carbon, silicon, silicates, ceramics, minerals and polymers (silicone) (Figure 1). Attached to the advantage of the porous materials, micro, meso and macro channels can be coated with metallic nanoparticles to further improve their biocompatibility properties. Most researches have been aimed at providing porous materials, channels and walls with surface functionality focused primarily on silica-based materials, where the structure can be functionalized with organic phases, aliphatic or aromatic groups to interact with metals [4] (Figure 2). The porous materials could be made with the use of organic compounds that could act as templates or pore-forming agents, through a versatile non-surfactant route (sol-gel process) in which non-surfactant organic compounds such as D-glucose, D-maltose, dibenzoyl-L-tartaric acid, ascorbic acid, etc., were used and then removed by solvent extraction [5]. Another way is to use surfactants; these are widely used structure-directing agents that can control the growth of inorganic polymers into various sizes and shapes at the mesoscale (that is, the scale between atoms and macroscopic objects). Porous materials with various composition and structures, such as nanoparticles, nanowires, nanotubes and nanoporous materials, can be synthesized through simple solution chemistry using surfactant micelles to direct structure [6].
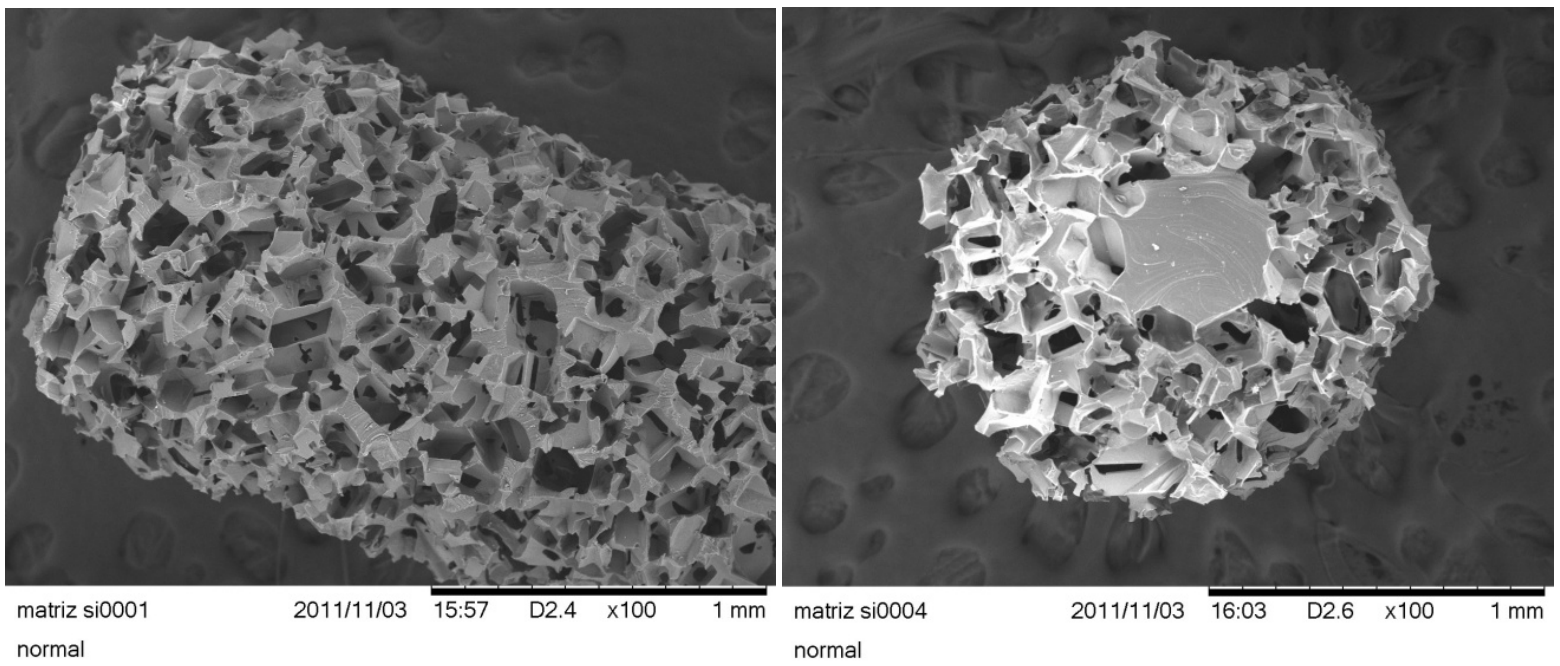

Figure 1. Porous silicon matrix made with the use of pore forming agents, SEM imaging 100×.

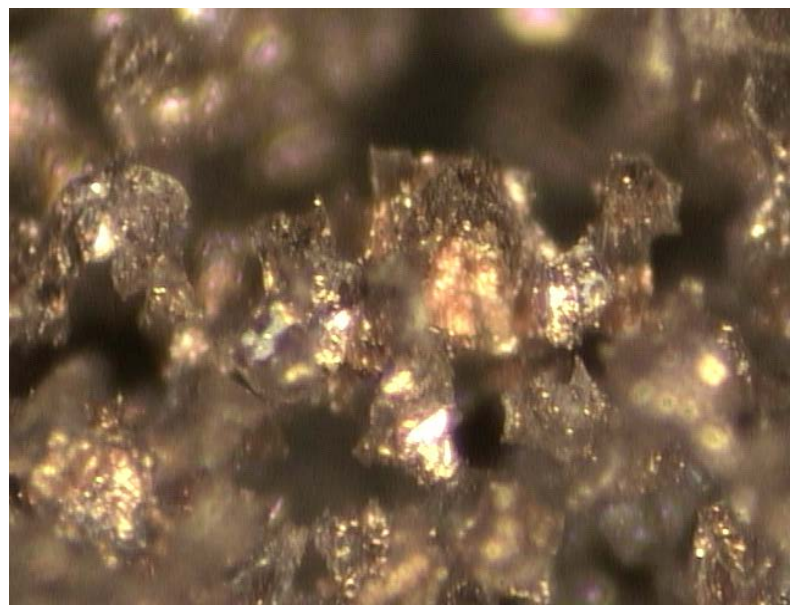

(a)

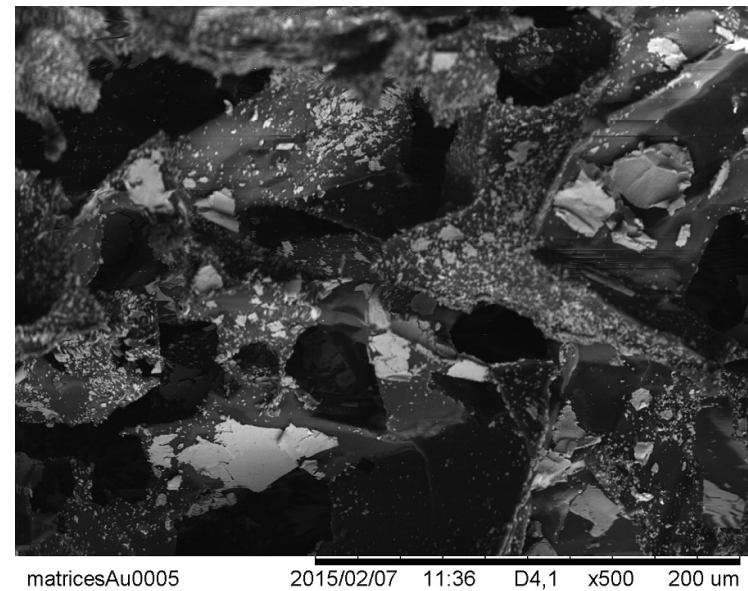

(b)

Figure 2. Porous silicon matrix after silver and gold deposition, (a) visual imaging 171×, (b) SEM imaging 500×. 
The application of porous materials is of great interest in various fields of medicine like:

o Implants;

o Drug delivery;

o Sensors;

o Membranes;

o Filters;

o Catheters;

o Valves;

o Prosthesis;

o Scaffolds;

0 etc.

\subsection{Porous Materials on Implants}

Biocompatible implants allow the human body to restore the biological and mechanical functions; therefore manage to increase the quality of life. Depending on the biomedical application, the implant has to withstand mechanical loads, while conducting a long term biological interaction with the surrounding biological tissue is promoted. Bulk properties of the implant are primarily responsible for the load bearing capacities, where the interaction with the surrounding tissue is governed by the implant surface [7] [8]. The properties of micro, meso and macroporous materials, based on the porous size, roughness, interconnected channels, functionalized surface included the pore walls can be able to induce tissue regeneration, these pores have been combined with their abilities to host (adsorption or absorption) and release specific biomolecules that promoted cell growing inside and over the porous surface. The possibility of locally deliver peptides and proteins is of great scientific importance because it opens new paths for design of implantable biomaterials than can promote bone formation, organs reconstruction, cell an tissue growing where needed [9].

Silica based mesoporous materials have been extensively proposed for different biomedical applications, such as ceramics for bone regeneration, because these materials exhibit high surface areas and pore volumes, tunable and narrow pore size distributions and these are easily functionalized. These properties allow the materials to act as host matrices for active molecules (drugs, peptides, proteins, etc.) [10].

The organic biomaterials with a porous structure have flexible nature with multiple control function that made them attractive for many advanced applications such as sensing, medical uses, and selective material conversion. Immobilization of biocompounds into organized structures paid much attention. However, most of them have difficulty in mechanical stability, and some of them have difficulty in material diffusion and unsuitability to make contact with artificial devices. Unlike these materials, inorganic porous materials have several advantages as media for biomaterial immobilization:

- They are mechanically much stronger;

- The diffusion of materials should be ensured in mesopore spaces;

- High flexibility in fabrication (shapes and sizes);

- The hybridized surface possibility on mesoporous materials increases the adsorption and absorption properties.

The combination of porous organic materials with inorganic materials is a developed research, and these products are actually base on the upstanding properties for specific applications. Direct substitution of component elements in original porous materials, with maintaining structural regularity, provided novel properties that could be applied on surgical implants [11].

The discovery of ordered porous materials and the wide range of used materials to obtain these, like silica, metal oxides, and sulfides open great field applications on nanoscience. Organic-inorganic hybrids play an important role in the design application; the siloxane and organosiloxane have been used to prepare hybrid porous materials with functional groups at their surface [12]. The advantage of porous materials (micro, meso and macro) and specifically on medical implants is bio mobility and integration, as well as a low incidence of removal, displacement and foreign body reaction.

Fibrovascular ingrowth into porous implants offers the potential for excellent prosthesis movement, and also reduces a chance of infection, exposure, and displacement, porous polyethylene, silicon, aluminum oxide have been used. Silicone is a material being used in medical science for variety of prosthetic applications, because of its 
excellent biocompatibility and low cost, some limitations of silicone are limited ingrowth but it can be improve with the introduction of functional groups or nanoparticles in its surface [13].

\subsection{Porous Materials for a Drug Delivery}

The applications of porous based materials as a potential drug delivery system have grown exponentially, in particular in the field of oral drug delivery vehicles to improve dissolution properties of hydrophobic drug molecules, and thus, there is a great need for drug delivery systems capable of improving the physicochemical properties [14]. Formulating poorly water soluble drugs is of great importance for human health, one way is through the functionalize of porous surface and another way is for a sol gel procedure, to encapsulate the drug in porous silica structure, this method is based on efficient solubilization of hydrophobic drugs in a surfactant micelles which act a template for the silica network [15] [16].

Porous silica and silicon have been studied with promising results on drug delivery systems [17]. The adsorption capacity and release properties of porous materials for drug molecules can be improved by functionalizing their surfaces with judiciously chosen organic groups; Functionalized porous materials could be containing various types of organic groups via a co-condensation synthetic method from organosilanes and by post-grafting onto mesoporous silica [18]. On some porous materials like zeolites, the surface modification can occur inside the pore channels or on the external surface, depending on the size of the organometallic complex or functional group. When the modification occurs on the external surface, it results in a decrease of the pore entrance, which lead in turn to a modification of the sorption properties of the micro or mesoporous matrix, by decreasing the rate of the adsorption (mainly by kinetic control) [19]. This interactions between the matrix and the adsorbed molecule could be study by the combination of the two techniques provides the first direct proof, the molecule diffusion pathway through the pore system correlates with the orientation, the influence of specific structural features of the host on the diffusion behavior of the guest molecules can be clearly seen with a single fluorescent dye molecule application, how it changes speed in channel structure, and how it bounces off domain boundary. The combination of electron microscopy and optical single-molecule opens up new ways of understanding the interactions of host and guest [20].

The continuous researching labor on porous materials developed a variety of approaches for many applications of this material in the industrial area and most important on the medical field. Metal-organic (hybrids) frameworks also show promising properties, although this structure combine with organic functionalities can enhance the range of applications to more realistic conditions than pure inorganic mesoporous materials. Pure inorganic mesoporous materials have their intrinsic limitations lacking both functionalities and mechanical strength [21].

A key issue in biotechnology is the stabilization of biological active molecules under non-physiological conditions, as their tendency to lose activity in artificial environments. Immobilization onto inert inorganic materials may cope with stability and compatibility problems, thus favoring advance in technological applications. The success of such a strategy depends on proper selection of the hybrid materials that can support or host the bioactive components while maintaining its activity and selectivity [22] [23]. Some examples of this application are:

- Adsorption profiles of insulin on porous materials and release profiles of insulin entrapped on folded sheet mesoporous silica were study with successful results. The amount of adsorbed insulin, estimate by protein assay increased with an increase in the pore sizes, and the release of the drug could be controlled by varying the pore size, also the encapsulation of the insulin may have potential to avoid enzymatic degradation [24].

- Porous silica impregnated with anticancer drug (doxorubicin) is another research area, sorption and desorption of the drug is investigated. Dexorubicin is an anthracycline cytostatic antibiotic with against a wide range of human cancers application, but its use is limited by the toxicity side effects, to reduce this limitation and improve delivery to tumor site, various targeted drug delivery systems such, liposomes, nanoparticles, polymer micelles and porous materials are evaluate [25].

- Metalloproteins has attracted considerable attention for applications in mediator-free biosensors. Hemoglobin, a heme protein that stores and transports oxygen, consists of four polypeptides with a redox behavior, it is a large protein matrix, that makes it difficult to establish direct electron transfer between hemoglobin and conventional electrodes, but the immobilization of this redox protein on mesoporous material can be avoid this issue, and it represents a promising applications on a biological fields, for a novel biosensors development [26]. 
- The porous materials functionalized with mercaptopropyl and vinyl groups showed greater adsorption capacity and good release behavior for rhodamine 6G molecules. On the other hand, the porous matrices that contain amine functional groups showed greater adsorption capacity and better release properties for ibuprofen molecules. Improved adsorption and release properties of porous materials by simple changes of functional groups on the porous channels walls are the key for drug delivery systems [27].

Poor aqueous solubility is acknowledged as one of the major barriers in the development of novel pharmaceutical agents. More than $40 \%$ of newly identified lead compounds are lipophilic, resulting in poor pharmacokinetic properties, insufficient dissolution, unfavorable drug-drug interactions, poor drug absorption and bioavailability, which leads to need high dosing to reach therapeutic levels in blood. Organic and inorganic nanomaterials are currently being studied as drug delivery vehicles, to achieve supersaturation in the gastro-intestinal environment. Mesoporous materials have emerged as suitable excipients with a complete set of desirable properties in comparison to traditional formulation strategies relay on administering poor water soluble drugs as a solution or emulsion in present of surfactants or complex agents [28].

Implants for topical delivery of drugs like corticosteroids or antibiotics are a novel approach to manage postsurgical complications associated to inflammation or infections. A controlled release drug delivery consists of two basic components, that is, a biologically active agent and the drug carrier, the system has numerous advantages over conventional forms of dosage:

- Maintaining the patient blood level;

- Minimizing deleterious side effects;

- Prolonging efficiency time;

- Heightening bioavailability;

- Protecting sensitive drugs from enzymatic or acidic degradation;

- Helps to maintain the drug on biological fluids within a desired therapeutic range;

- Deliver the drug locally to specific site;

- Avoid possible toxicity;

- Increase patient compliance and comfort;

- Decreasing the dosing, and frequency [29].

Silicone rubber (PDMS) is well-known polymeric material frequently used to develop drug delivery devices, along with its customary medical applications such as parts in cardiac pacemaker leads, mammary prostheses, drainage implants in glaucoma, temporary skin substitutes, blood oxygenators, medical adhesives, finger joints, and catheters [30]. Nanocarries have been demonstrated to have potential in improving the safety profile and therapeutic efficiency of current therapies for autoimmune diseases, particular for those with potent but toxic compounds. In inflammatory arthritis, nanocarries infiltrate the cells at the inflammation sites, resulting in the targeting of the affected area. It is important to note that most of the drugs used in nanomedecine development are approved by FDA; this will accelerate clinical translation compared with developing a new drug [31].

The porous materials have diverse range of applications such medical imaging, drug delivery, implants, or sensors. The used of porous silicone on the medical field achieving a sharp technologies that can increase the quality human life, though surface modification this porous material can combine hydrophobicity properties inside the pores, silicone surface could be obtain with an etching process and later it is possible to introduce and join reactive functional groups on the porous silicon surface to obtain special properties. The different internal surfaces on porous materials impart different physical properties, a surface modified with the hydrophobic functional groups avoids the water ingress inside the pores, in contrast if the functional groups are hydrophilic the water passes, and the material is filled with water [32].

\subsection{Porous Materials on Medical Imaging}

Noninvasive imagine techniques are of foremost relevance diagnostics, diverse imaging modalities are available with porous technology application, such magnetic resonance imaging (MRI) and computed tomography (CT), to those that provide specific molecular imaging such as positron electron tomography (PET) and optical fluorescence.

Contrast agents for a medical imaging diagnostic have to fulfill important prerequisites:

1) Forming stable colloidal solution in vitro and in vivo environments;

2) Chemical stability under physiological conditions; 
3) Exhibiting limited nonspecific binding to avoid macrophagocytic system uptake;

4) Having programmed clearance mechanisms;

5) Showing high sensitivity and selectivity for target;

6) Good image contrast;

7) Having relatively long circulation time in the blood if administered intravenously.

In this sense porous material based contrast agents are promising platforms to meet the aforementioned prerequisites [33].

Many nanosized particles systems are being developed as intravascular carriers to increase the levels of therapeutic agents delivered to targets, the surface of these carriers is often functionalized with biological recognition molecules for specific barriers in the body that prevent these carriers from localizing at their targets at sufficiently high therapeutic concentrations. Multistage delivery system can carry, release over time and deliver two types of nanoparticles into primary endothelial cells, multistage system is based on biodegradable and biocompatible porous silicone particles the included therapeutic drug and imaging contrast agents [34].

Nanoparticles have become more prevalent in reports of novel contrast agents, especially for molecular imaging, the detection of cellular process. The advantages of nanoparticles include their potency to generate contrast, the ease of integrating multiple properties, lengthy circulation times, and the possibility to include high payloads. With this technology and the used of micropores, mesopores and macropores materials, more sophisticated examples of contrast agents have been reported, such as paramagnetic, macrophage, targeted quantum dots, MRI visible micro-emulsions, etc. the used of this technology is producing a greater knowledge of diseases process and effects of therapy [35].

\subsection{Porous Materials on Biological Sensors}

The use of computational models in the design of sensors is an increasing area of interest, in their design two main issues have be taken into account to model the behavior: on one hand the interaction between recognition agent and analyte and the signal transduction, in this work porous membrane are explore with a successful results. Micro, meso and macroporous systems have been presented in different formats as a potential tool or framework for detection analytes or by simple using a common property to all molecules or recognition agents (label sensors) [36]. Porous materials are finding increasing utility in sensing applications. This application can benefit from a surface area that may exceed $1000 \mathrm{~m}^{2} / \mathrm{g}$ and fast diffusion of analytes through a porous structure, these factors contribute to the selectivity and sensitivity of a sensor application response, chemical functionalization of this porous materials through grafting, co-condensation, and adsorption are also address to improve this properties [37].

Many studies have been carried out for early diagnosis of complex diseases by finding accurate, robust and stable biomarkers specific to diseases. In particular, recent rapid advance of high-throughput technologies provides unprecedented rich information to characterize various disease genotypes and phenotypes, also a new acknowledge about nanoscience, micro and mesoporous materials accelerates significantly the study of biomarkers from both theoretical and clinical perspectives [38]. Biological active peptides such as peptide hormones and cytokines play pivotal roles in human health and diseases, therefore the comprehensive analysis in complex biological mixtures can contribute to a better understanding of the biochemical functions of peptides, as well to the develop of biomarkers. Resent research on porous materials has shown great potential to peptide enrichment include and accurate and controllable molecular weight cut off to separate peptides from interfering proteins, a strong retention that permit the peptide capture for the study and future applications [39].

Porous materials were utilized to immobilize enzymes, their huge area, modifiable surface, and restricted spaces generated synergistic effects that enhance enzyme stability, improve product selectivity, and facilitate separation and reuse of enzymes. The immobilized property of molecules and enzymes on pores materials can be applied as viable biocatalysts and pharmaceutical industries on medical applications for a drug delivery, imaging, sensors, implants, etc [40]. These materials offer superior ways to maintain biological activity and improve the sensitivity of biosensors. With the development of nanoscience and nanotechnology, the combination porous materials and enzymes could lead to the development of multi-functional assembly systems with novel electronic properties. Such coupling of high-sensitivity and high-stability capabilities allows electrochemical biosensors to rival the most advanced electrochemical and optical protocols in bioassays [41].

The poly dimethylsiloxane (PDMS) have gaining popularity base on its possibility to made micro and meso- 
porous frameworks that can stabilize functional groups and nanoparticles with a special properties the can be used for biological applications, their non-toxicity, easy fabrication, scalability, optical transparency, and low cost potentially all recently research. The surface modification methods developed for a micro and mesoporous PDMS material can improve the repelling properties (hydrophobicity) and open the way too easily and strongly interactions between the base material and biological molecules, organs and tissues, this improvement on PDMS surface permits the porous PDMS use on biological analysis, such as biomolecule separation, immunoassay, cell culture and DNA hybridization [42].

The currently, standard methods for diseases detection require expensive and bulky instruments, and they have associated delays, and lack of selectivity. In order to solve these problems, recent efforts have been directed toward the development of biological methods of detection combined with physical transducers (biosensors). In particular, amperometric biosensors with an immobilized enzyme on a porous matrix can offer high sensitivity, high selectivity and short response time without requiring a complicated instrumentation [43]. Base on porous selectivity a novel proposed strategy for the insolation and analysis of multi-phosphopeptides on $\mathrm{TiO}_{2}$ porous silica base materials was developed and showed highly selective and sensitive properties. Protein phosphorylation as one of the most important post-translational modifications in mammalian cell regulates numerous biological process [44].

Nanoscience and nanotechnology produce a revolution on the medical field and biological science because application of unique characteristics from the combine of micro, meso, macroporous and nanoparticles has been expanding on animal and human help procedures like sensing, imaging, cell targeting, drug delivery, etc, because cells can readily uptake nanoparticles from these systems.

Micro and mesoporous materials are high performance materials in their own right, for instance as catalysts highly selective absorbents, but addition they provide excellent opportunities for the creation of materials with additional functionality. Their pore system can be used to introduce molecules or particles that are stabilized by the solid framework [45].

Most of porous materials are mainly microporous and this makes them well-suited for many applications on a medical industry. However, there are numerous potential applications in which materials with pores would be advantageous. Therefore, the control of the textural and structural properties of porous materials is one of the main subjects because they are very important for certain applications [46].

\section{Conclusions}

The use of micro, meso and macroporous material can be a very practical approach to reaching several goals in current medical applications like surgical implants, drug delivery, medical imaging, biological sensors, and others. Their performance under ambient conditions, mechanically and thermally strong under harsh conditions is the key. The hybridization of supporting materials and the combination of polymers and inorganic materials with a porous structure have been proposed to attain both biological and mechanical requirements.

Porous materials have been either organic or inorganic, inorganic solids such as silicas and metal oxides are usually crystalline but organic solids such as activated carbon is amorphous or partially ordered like graphite or diamond. In contrast ordered porous, organic-inorganic hybrid materials such as organosilicas, combine the respective beneficial characters of organic and inorganic components but also exhibit exceptional properties with enormous opportunities for medical applications in drug delivery, imaging and other medical applications [47].

Great progresses in structure control and functionalization of porous materials have been achieved for biotechnological and biomedical applications. Silica-based controlled drug delivery systems, magnetism and/or luminescence, functionalized systems, which integrated targeting and tracking abilities of molecules, stimuli-responsive controlled release systems that are able to respond to environmental changes, such as $\mathrm{pH}$, redox potential, temperature, photoirradiation, etc, have been achieved [48].

The porous materials, their size control, density, channel connectivity, the roughness and functionalization of the surface are the keys for an enormous and future application, the medicine field is included in this movement, the new medical device design is compared with a building construction where you can control the room size, quantity, connected aisles and the materials for the walls, all of these, based on the used or application that you can give.

The researchers will be the nano-architects of the future porous structures that support new developments and applications on the medical field. 


\section{References}

[1] Kuschel, A. and Polarz, S. (2008) Organosilica Materials with Bridging Phenyl Derivatives Incorporated into the Surfaces of Mesoporous Solids. Advanced Functional Materials, 18, 1272-1280. http://dx.doi.org/10.1002/adfm.200701252

[2] Mitra, A., Vázquez-Vázquez, C., López-Quintela, L., Bidyut, P. and Bhaumik, A. (2010) Soft-Templating Approach for the Synthesis of High Surface Area and Superparamagnetic Mesoporous Iron Oxide Material. Microporous and Mesoporous Materials, 131, 373-377. http://dx.doi.org/10.1016/j.micromeso.2010.01.017

[3] Park, J., Jung, D., Myung, S., Kim, S., Moon, W., Shin, Ch. and Seo, G. (2008) Preparation of Mesoporous Materials with Adjustable Pore Size Using Anionic and Cationic Surfactants. Microporous and Mesoporous Materials, 112, 458466. http://dx.doi.org/10.1016/j.micromeso.2007.10.023

[4] Ozin, G. and Arsenault, A. (2005) Nanochemistry A Chemical Approach to Nanomaterials. ESC Publishing, Canada, 396-417. http://dx.doi.org/10.1002/cjoc.20000180507

[5] Pang, J., Qiu, K. and Wei, Y. (2000) Synthesis of Mesoporous Silica Materials with Ascorbic Acid as Template via Sol-Gel Process. Chinese Journal of Chemistry, 18, 693-697. http://dx.doi.org/10.1002/cjoc.20000180507

[6] Ryoo, R. (2009) A Tricontinuous Mesoporous System. Nature Chemistry, 1, 105-106. http://dx.doi.org/10.1038/nchem.190

[7] Zhang, S., Ed. (2011) Biological and Biomedical Coating Handbook Applications. CRC Press Taylor \& Francis Group, Boca Raton, 378-426.

[8] Kretlow, J. and Mikos, A. (2008) From Material to Tissue: Biomaterial Development, Scaffold Fabrication, and Tissue Engineering. AIChE Journal, 54, 3048-3067. http://dx.doi.org/10.1002/aic.11610

[9] Balas, F., Manzano, M., Colilla, M. and Vallet-Regi, M. (2008) L-Trp Adsorption in to Silica Mesoporous Materials to Promote Bone Formation. Acta Biomaterilia, 4, 514-522. http://dx.doi.org/10.1016/j.actbio.2007.11.009

[10] Izquierdo-Barba, I., Sánchez-Salcedo, S., Colilla, M., Feito, M., Ramírez-Santillán, C., Portolés, M. and Vallet-Regí, M. (2011) Inhibition of Bacterial Adhesion on Biocompatible Zwitterionic SBA-15 Mesoporous Materials. Acta Biomaterialia, 7, 2977-2985. http://dx.doi.org/10.1016/j.actbio.2011.03.005

[11] Vinu, A., Mori, T. and Ariga, K. (2006) New Families of Mesoporous Materials. Science and Technology of Advanced Materials, 7, 753-771. http://dx.doi.org/10.1016/j.stam.2006.10.007

[12] Zhang, W.H., Lu, X.B., Xiu, J.H., Hua, Z.L., Zhang, L.X., Robertson, M., Shi, J.L., Yan, D.S. and Holmes, J.D. (2004) Synthesis and Characterization of Biofunctionalized Ordered Mesoporous Materials. Advanced Functional Materials, 14, 544-552. http://dx.doi.org/10.1002/adfm.200305001

[13] Son, J.H., Kim, C.S. and Yang, J.W. (2012) Comparison of Experimental Porous Silicone Implants and Porous Silicone Implants. Graefe's Archive for Clinical and Experimental Ophthalmology, 250, 879-885. http://dx.doi.org/10.1007/s00417-011-1902-7

[14] Ayral, A., Coq, B. and Fajula, F. (2011) Special Issue of Microporous and Mesoporous Material. Microporous and Mesoporous Materials, 140, 1. http://dx.doi.org/10.1016/j.micromeso.2010.12.006

[15] Tourne-Peteilh, C., Begu, S., Lerner, D., Galarneau, A., Lafont, U. and Devoisselle, J. (2012) Sol-Gel One-Pot Synthesis in Soft Conditions of Mesoporous Silica Material Ready for Drug Delivery System. Journal of Sol-Gel Science and Technology, 61, 455-462. http://dx.doi.org/10.1007/s10971-011-2646-x

[16] Fu, T.M., Lu, J., Guo, L.W., Zhang, L.J., Cai, X.P. and Zhu, H.X. (2012) Improving Bioavailability of Silybin by Inclusion into SBA-15 Mesoporous Silica Materials. Journal of Nanoscience and Nanotechnology, 12, 3997-4006. http://dx.doi.org/10.1166/jnn.2012.5826

[17] Kinnari, P., Makila, E., Heikkila, T., Salonen, J., Hirvonen, J. and Santos, H. (2011) Comparison of Mesoporous Silicon and Non-Ordered Mesoporous Silica Materials as Drug Carriers for Intraconazole. International Journal of Pharmaceutics, 414, 148-156. http://dx.doi.org/10.1016/j.ijpharm.2011.05.021

[18] Wang, G., Otuonye, A., Blair, E., Denton, K., Tao, Z.M. and Asefa, T. (2009) Functionalized Mesoporous Materials for Adsorption and Release of Different Drug Molecules: A Comparative Study. Journal of Solid State Chemistry, 182, 1649-1660. http://dx.doi.org/10.1016/j.jssc.2009.03.034

[19] Lefebvre, F., Putaj, P., Basset, J., Wang, X.X. and Fu, X.Z. (2010) Modification of the Adsorption and Catalytic Properties of Micro and Mesoporous Materials by Reaction with Organometallic Complexes. Science China Chemistry, 53, 1862-1869. http://dx.doi.org/10.1007/s11426-010-4068-y

[20] Zürner, A., Kirstein, J., Döblinger, M., Bräuchle, C. and Bein, T. (2007) Visualizing Single-Molecule Diffusion in Mesoporous Materials. Nature, 450, 705-709. http://dx.doi.org/10.1038/nature06398

[21] Qin, Y.C., Ren, H.B., Zhu, F.H., Zhang, L., Shang, C.W., Wei, Z.J. and Luo, M.M. (2011) Preparation of POSS-Based Organic-Inorganic Hybrid Mesoporous Materials Networks through Schiff Base Chemistry. European Polymer Jour- 
nal, 47, 853-860. http://dx.doi.org/10.1016/j.eurpolymj.2011.02.024

[22] Giussani, L., Fois, E., Gianotti, E., Tabacchi, G., Gamba, A. and Coluccia, S. (2010) On the Compatibility Criteria for Protein Encapsulation inside Mesoporous Materials. ChemPhysChem, 11, 1757-1762. http://dx.doi.org/10.1002/cphc.200901038

[23] Toru, O., Masahiro, T., Megumi, H. and Katsuya, K., (2012) Binding Activity of Avidin to the Biotin within Mesoporous Silica Materials for Bioanalytical Applications. Analytical Biochemistry, 425, 1-9. http://dx.doi.org/10.1016/j.ab.2012.02.037

[24] Tozuka, Y., Sugiyama, E. and Takeuchi, H. (2010) Release Profile of Insulin Entrapped on Mesoporous Materials by Freeze-Thaw Method. International Journal of Pharmaceutics, 386, 172-177. http://dx.doi.org/10.1016/j.ijpharm.2009.11.012

[25] Prokopowicz, M. and Przyjazny, A. (2007) Sybthesis of Sol-Gel Mesoporoues Silica Materials Providing a Slow Release of Doxorubicin. Journal of Microencapsulation, 24, 694-713. http://dx.doi.org/10.1080/02652040701547658

[26] Nie, D.X., Liang, Y., Zhou, T.S., Li, X.H., Shi, G.Y. and Jin, L.T. (2010) Electrochemistry and Electrcatalytic of Hemoglobin on FDU-15-Pt Mesoporous Materials. Bioelectrochemistry, 79, 248-253. http://dx.doi.org/10.1016/j.bioelechem.2009.12.008

[27] Asefa, T., Otuonye, A., Wang, G., Blair, E., Vathyam, R. and Denton, K. (2009) Controlling Adsorption and Release of Drug and Small Molecules by Organic Functional of Mesoporous Material. Adsorption, 15, 287-299. http://dx.doi.org/10.1007/s10450-009-9176-7

[28] Xia, X., Zhou, C.F., Ballell, L. and Garcia-Bennett, A. (2012) In Vivo Enhancement in Bioavailability of Atazanavir in the Presence of Proton-Pump Inhibitors using Mesoporous Materials. ChemMedChem, 7, 43-48. http://dx.doi.org/10.1002/cmdc.201100500

[29] Qu, F.Y., Zhu, G.S., Lin, H.M., Zhang, W.W., Sun, J.Y., Li, S.G. and Qiu, S.L. (2006) A Controlled Release of Ibuprofen by Systematically Tailoring the Morphology of Mesoporous Silica Materials. Journal of Solid State Chemistry, 179, 2027-2035. http://dx.doi.org/10.1016/j.jssc.2006.04.002

[30] Nemati, P., Imani, M., Farahmandghavi, F., Mirzadeh, H., Marzban-Rad, E. and Nasrabadi, A. (2013) Artificial Neural Networks for Bilateral Prediction of Formulation Parameters and Drug Release Profiles from Cochlear Implant Coatings Fabricated as Porous Monolithic Devices Base on Silicone Rubber. Journal of Pharmacy and Pharmacology, 66, 624-638. http://dx.doi.org/10.1111/jphp.12187

[31] Yuan, F., Thiele, G. and Wang, D. (2011) Nanomedicine Development for Autoimmune Diseases. Drug Development Research, 72, 703-716. http://dx.doi.org/10.1002/ddr.20479

[32] Kilian, K., Bocking, T., Gaus, K. and Gooding, J. (2008) Introducing Distinctly Different Chemical Functionalities onto the Internal and External Surfaces of Masoporous Materials. Angewandte Chemie, 120, 2737-2739. http://dx.doi.org/10.1002/ange.200704784

[33] Regí, M., García, M. and Colilla, M. (2013) Biomedical Applications of Mesoporous Ceramics, Drug Delivery, Smart Materials and Bone Tissue Engineering. CRC Press, New York, 149-155.

[34] Tasciotti, E., Liu, X.W., Bhavane, R., Plant, K., Leonard, A., Prince, B., Cheng, M., Decuzzi. P., Tour, J., Robertson, F. and Ferrari, M. (2008) Mesoporous Silicon Particles as a Multistage Delivery System for Imaging and Therapeutic Applications. Nature Nanotechnology, 3, 151-157. http://dx.doi.org/10.1038/nnano.2008.34

[35] Cormode, D., Skajaa, T., Fayad, Z. and Mulder, W. (2009) Nanotechnology in Medical Imaging: Probe Design and Applications. Arteriosclerosis, Thrombosis, and Vascular Biology, 29, 992-1000. http://dx.doi.org/10.1161/ATVBAHA.108.165506

[36] Peinetti, A., González, G. and Battaclini, F. (2010) Modeling the Electrochemical Response of Mesoporous Materials Toward Its Application to Biomolecular Detection. Electrochemical, 22, 1329-1336. http://dx.doi.org/10.1002/elan.200900572

[37] Melde, B. and Johnson, B. (2010) Mesoporous Materials in Sensing Morphology and Functionality at the Meso-Interface. Analytical and Bioanalytical Chemistry, 398, 1565-1573. http://dx.doi.org/10.1007/s00216-010-3688-6

[38] Liu, R., Wang, X.D., Aihara, K. and Chen, L.N. (2014) Early Diagnosis of Complex Diseases by Molecular Biomarkers, Network Biomarkers, and Dynamical Network Biomarkers. Medicinal Research Reviews, 34, 455-478. http://dx.doi.org/10.1002/med.21293

[39] Li, F., Dever, B., Zhang, H.Q., Li, X.F. and Le, C. (2012) Mesoporous Materials in Peptidome Analysis. Angewandte Chemie, 51, 3518-1519. http://dx.doi.org/10.1002/anie.201107849

[40] Lee, C.H., Lin, T.S. and Mou, C.Y. (2009) Mesoporous Materials for Encapsulating Enzymes. Nano Today, 4, $165-179$. http://dx.doi.org/10.1016/j.nantod.2009.02.001

[41] Hasanzadeh, M., Shadjou, N., De la Guardia, M., Eskandani, M. and Shekhzadeh, P. (2012) Mesoporous Silica-Base 
Materials for Use in Biosensors. Trends in Analitical Chemistry, 33, 117-129. http://dx.doi.org/10.1016/j.trac.2011.10.011

[42] Tu, Q., Wang, J.C., Zhang, Y., Liu, R., Liu, W., Ren, L., Shen, S., Xu, L. and Wang, J. (2012) Surface Modification of Poly(dimethylsiloxane) and Its Applications in Microfluidics-Base Biological Analysis. Analytical Chemistry, 31, 177-192.

[43] Shimomura, T., Itoh, T., Sumiya, T., Mizukami, F. and Ono, M. (2008) Electrochemical Biosensor for the Detection of Formaldehyde Base on Enzyme Immobilization in Mesoporous Silica Materials. Sensors and Actuators B: Chemical, 135, 268-275. http://dx.doi.org/10.1016/j.snb.2008.08.025

[44] Guo, G.C., Wan, J.J., Qian, K., Yu, C.Z., Kong, J.L., Yang, P.Y. and Liu, B.H. (2011) $\mathrm{TiO}_{2}$-Functionalized Mesoporous Materials for Sensitive Analysis of Multi-Phosphopeptides. Science China Chemistry, 54, 1327-1333. http://dx.doi.org/10.1007/s11426-011-4344-5

[45] Schüth, F. and Schmidt, W. (2002) Microporous and Mesoporous Materials. Advanced Materials, 14, 629-638. http://dx.doi.org/10.1002/1521-4095(20020503)14:9<629::AID-ADMA629>3.0.CO;2-B

[46] Calvillo, L., Celorrio, V., Moliner, R., Cabot, P., Esparbe, I. and Lazaro, M. (2008) Control of Textural Properties of Ordered Mesoporous Materials. Microporous and Mesoporous Materials, 116, 292-298. http://dx.doi.org/10.1016/j.micromeso.2008.04.015

[47] Xuan, W.M., Zhu, C.F., Liu, Y. and Cui, Y. (2012) Mesoporous Metal-Organic Framework Materials. Chemical Society Reviews, 41, 1677-1691. http://dx.doi.org/10.1039/C1CS15196G

[48] Yang, P.P., Gai, S.L. and Lin, J. (2012) Functionalized Mesoporous Silica Materials for Controlled Drug Delivery. Chemical Society Reviews, 41, 3679-3698. http://dx.doi.org/10.1039/c2cs15308d 